\title{
Student incivility: Nursing faculty lived experience
}

\author{
Elizabeth Ann Sprunk ${ }^{1}$, Kathleen B. LaSala², Vicki L. Wilson ${ }^{3}$ \\ 1. Mercy College of Ohio, Toledo, USA. 2. University of South Carolina, Columbia, USA. 3. University of Northern Colorado, \\ Greeley, USA. \\ Correspondence: Elizabeth Ann Sprunk. Address: Mercy College of Ohio, Toledo, USA. \\ Email: elizabeth.sprunk@mercycollege.edu
}

Received: March 9, 2014

DOI : 10.5430/jnep.v4n9p1

\author{
Accepted: May 20, 2014 \\ Online Published: June 22, 2014 \\ URL: http://dx.doi.org/10.5430/jnep.v4n9p1
}

\begin{abstract}
Student incivility against nursing faculty is recognized as an area of increased concern in nursing education. The negative experience that this may potentially have on nursing faculty is disturbing. The purpose of this study was to elicit an understanding of the experiences and impact nursing faculty encountered with nursing student incivility using a phenomenological research design. Twelve nursing faculty members from seven mid-western universities provided rich descriptions of their experiences with student incivility. Colaizzi's analysis method was used to create clusters that resulted in six identified themes, including: (a) Faculty are subjected to a variety of unacceptable student behaviors; (b) Dealing with incivility is time consuming; (c) An aftermath of incivility can tarnished one's reputation; (d) Support from others is beneficial; (e) Can cause harm to one's health and well-being; and (f) May result in questioning the future. Findings support the view that nursing student incivility is becoming more widespread on college campuses and can have devastating effects on nursing faculty members. This information strongly suggests the importance of identifying contributing factors of incivility present in nursing students and implementing new and more effective policies and strategies to address and prevent this increasingly prevalent problem.
\end{abstract}

\section{Key words}

Incivility, Nursing students, Nursing faculty, Experience, Multifaceted tribulations, Policies

\section{I ntroduction}

Workplace incivility is a prevalent problem in today's society, and the nursing academic work environment is not immune to this phenomenon. Incivility is often described as any type of action or conduct that disrupts the work, social, personal, or educational environment ${ }^{[1]}$. Workplace incivility may cause negative physical and psychological effects on an individual, such as stress, anxiety, illness, job dissatisfaction, and absenteeism. In addition, workplace incivility may negatively impact retention and recruitment of qualified workers ${ }^{[2-5]}$. One of the most drastic effects of incivility is violent behavior, of which nursing education has experienced ${ }^{[6]}$. Consistent with the broad definition of incivility, academic incivility is broadly described as any action, or conduct that disrupts the teaching or learning milieu ${ }^{[1]}$, including the classroom, clinical, and online settings. Incivility against nursing faculty by nursing students is recognized as an area of increased concern in nursing education and has been reported in all areas of nursing education ${ }^{[7]}$. The negative potential impacts on nursing faculty are alarming. A threat to the psychological and physical well-being of nursing faculty is a potential consequence of nursing student incivility, and may be detrimental to the recruitment and retention of faculty, an academic 
area that is already experiencing a large shortage of qualified nurse educators ${ }^{[8,9]}$. Academic incivility may negatively affect the student-teacher relationship, as well as the teaching-learning process ${ }^{[10,11]}$. Clark ${ }^{[12]}$ noted that incivility occurs on a continuum, ranging from mild, irritating, and annoying behaviors to severe, aggressive, threatening, and potentially violent behaviors. Stress associated with nursing education has been identified as a major cause of both nursing student and faculty incivility ${ }^{[10]}$. When people experience stress, uncivil behaviors become more common, which in turn can escalate into violent behavior if not constrained ${ }^{[1]}$.

There is a need for nursing research to illuminate the character of the lived experience through the eyes of nursing faculty, including the potential consequences. There is a lack of empirical studies that describe faculty experiences and reactions to nursing student incivility. The purpose of this phenomenological study is to describe and understand the experiences and consequences nursing faculty have had with nursing student incivility at a college or university. Findings may help bring awareness of the issues and guide the development of policies, procedures, and interventions in the academic setting to deal with and prevent nursing student incivility. The overarching research question that guided this study was: What is the lived experience of a nursing faculty member who has experienced nursing student incivility? Sub questions included the following: What are faculty responses and reactions to nursing student incivility; What are consequences suffered by faculty related to nursing student incivility experiences; What is it like for nursing faculty to teach nursing students after an experience of incivility?

\section{Relevant scholarship/ literature}

Several definitions of academic incivility in various contexts have been noted in the nursing literature. Overall, the definitions are similar. These definitions point out that incivility not only disrupts the teaching-learning process, but also negatively impacts the well-being of an individual and the relationship between individuals.

The outcomes of incivility can include elevated stress levels, headache, inability to sleep, and a weakened immune system, leading to illness. Common examples of emotional effects are erosion of self-esteem, self-doubt, anxiety, and depression. In addition, incivility may lead to impairment of cognition, resulting in an inability to concentrate or learn. Incivility may lead to behavioral changes, such as withdrawal, retaliation, and potential violence ${ }^{[6,8,10,13]}$. Incivility also weakens personal relationships. In the academic setting, this could lead to impaired relationships between students and faculty, as well as between faculty members. In the academic setting, incivility may also impact recruitment and retention of faculty and students, hinder job satisfaction, increase absenteeism of faculty and students, and interfere with communication and collaboration $^{[2,3,10,11]}$.

The rigors of nursing education are stressful, often leading to physical and psychological symptoms, behavioral changes including incivility, as well as impeding the learning process ${ }^{[14-16]}$. Examples of stress include the clinical experience expectations, large amount of academic work in the nursing theory classes, time management and financial pressures, and strain on personal relationships ${ }^{[14,17]}$. The need to work full-time or part-time in addition to pursuing the rigors of a demanding nursing program are frequent and persistent stressors that students are faced with, and often lack the ability to cope effectively with. The fear of failure, combined with nursing faculty who frequently demonstrate a lack of caring, add to the already stressful experience nursing students face and leads to a struggle between nursing faculty and students. Robertson ${ }^{[18]}$ noted that this struggle devalues and diminishes the educational experience for both faculty and students, and, in turn, may lead to frustration and uncivil behaviors. Individually, each problem is manageable, but it is the compounding effect of numerous issues and factors that aggravate the situation, leading to incivility ${ }^{[15,16,18-21]}$.

The problem of incivility in nursing education as viewed from the perspective of the nursing student and the nursing faculty member has been perceived to be a moderate problem ${ }^{[6,8,22]}$. Several nurse researchers investigating incivility in nursing education have indicated that students were more likely to engage in uncivil behavior than were faculty. The most commonly noted student negative behaviors noted by these researchers were arriving late for class, leaving class early, cutting class, and not paying attention in class. Other uncivil behaviors of students noted were cell phone usage, sleeping in 
class, and cheating. The most frequent faculty behaviors noted were belittling or humiliating students, being distant or cold, being inflexible, and being unavailable outside of class ${ }^{[6,8,23]}$. Extreme behaviors of student incivility included vulgarity and harassing comments, challenging the faculty member's knowledge, taunting and disrespectful comments, and threats of physical violence ${ }^{[24]}$.

Academic incivility is not limited to expressions of uncivil behaviors between faculty and students. Faculty-to-faculty incivility is becoming an increased area of concern in nursing education. Nurse researchers investigating this phenomenon have found that uncivil behaviors between faculty members are common in the nursing academic environment ${ }^{[4,25-27]}$. Clark et al. ${ }^{[26]}$ found faculty-to-faculty incivility in nursing education to be a moderate to serious problem. Heinrich ${ }^{[4,27]}$ specifically addressed faculty-to-faculty incivility in a qualitative study. This author refers to faculty-to-faculty incivility as a joy stealing game that smothers relationships and deters the pursuit of knowledge and scholarship. Similarly, Clark ${ }^{[25]}$, in a recent national study, found that faculty-to-faculty incivility not only negatively impacts faculty members subjected to uncivil behaviors physically and psychologically, but also negatively impacts the educational organization. Facultyto-faculty incivility may result in increased absenteeism, decreased work performance, and high faculty turnover ${ }^{[25,26]}$.

After a review of the literature, this researcher found that very few qualitative studies have specifically addressed the lived experience of nursing faculty members who have experience nursing student incivility. This gap in the nursing literature identifies the necessity to investigate this phenomenon further. Continued research on this topic is needed to promote the importance of establishing civility in the nursing education environment. Civility, rooted in caring and mutual respect is essential for nursing education. Civility and ethical behaviors exhibited by students in nursing school are linked with civility and ethical behaviors exhibited in the nursing profession ${ }^{[1,28]}$. More research is needed on this topic to allow for a better understanding of this phenomenon. This in turn, may lead to the development of effective prevention strategies and help direct the development of policies and interventions in the academic setting to handle nursing student incivility in the nursing educational environment.

\section{Method}

A phenomenological design was used to examine the lived experience of faculty dealing with incivility from nursing students and its consequences. Congruent with the purpose of this study, Husserl's transcendental phenomenology was used to provide discovery of the understanding and valuing of the lived experience. This method seeks to describe individuals' common experiences of a particular phenomenon and provides for a common essence or meaning of the phenomenon to be described. This method helped the researcher illuminate and understand the lived experiences described by nursing faculty who participated in this study, and, in turn, helped to answer the research question ${ }^{[29-33]}$.

Approval was obtained from the Institutional Review Board prior to data collection. Participants were recruited from nursing faculty listings on websites at colleges and universities located in two Midwestern states and by word of mouth networking. Potential participants were sent an electronic message explaining the purpose of the study, benefits of the study, pertinent information regarding the study, as well as the researcher's contact information. The potential participants were asked to contact the researcher via telephone or e-mail indicating their interest in being a participant in the study. After making the initial contact with potential participants, the researcher then determined if the interested potential participants met the criteria for this study and had experienced nursing student incivility. The eligible participants were invited, verbally by the researcher, to participate in the study and had the option of whether to participate or not. The procedure of informed consent was followed to provide assurance of the rights of human subjects. Taking into consideration potential emotional responses of the participants, strategies to be followed in the event anxiety or distress occurred related to expressing or reliving the experience associated with nursing student incivility were outlined in the informed consent form. These included: (a) The researcher assessed the participant's level of comfort during the entire interview; (b) The researcher reminded the participant that he/she had the option to withdraw from the study at any time; and (c) A phone number to a local hospital and a local rescue center was provided prior to the interview commencing. 
The final purposive sample for this study consisted of 12 Caucasian women ages 39 to 65 ( $\bar{X}=55.8$ years), which is representative of the average age and race of nursing faculty in the two Midwestern states in which the study took place and in the United States. Ten were married, one divorced, and one single. All 12 were employed full-time at a college or university in one of two Midwestern states as nursing faculty members. Five taught mainly in the classroom, five taught in both the classroom and clinical, and two taught in the clinical setting only. Participants' hours of teaching per week ranged from 12 to 32 hours ( $\bar{X}=18.8$ hours/week). Years of teaching experience ranged from 2 to 35 years ( $\bar{X}=13.9$ years).

Descriptive data was collected via one-on-one interviews, lasting approximately 60 - 90 minutes. The interviews occurred either face-to-face in a quiet location of the participant's choice, such as their work office, or a quiet restaurant, or via telephone. The interview began with an open-ended statement: "Tell me about your experience with nursing student incivility.” The researcher used open-ended cues and prompts to obtain clarification and depth. An interview guide was used to provide direction for the discussion and a guide of issues that were covered during the interview, such as asking participants to describe their experiences with student incivility and how it affected them, and provide accounts of any physical or psychological consequences of incivility suffered, effects on the student-teacher relationship, and feelings about their future as a nurse educator. Follow up clarification was obtained as needed. Interviews were audio-taped and transcribed by the researcher. Audio tapes were erased after transcription was finalized. Transcriptions were stored electronically in a password protected file. Pseudonyms were used to protect confidentiality of participants. Demographic forms and participant responses were anonymous with no identifying information in the data summary.

Colaizzi’s ${ }^{[34]}$ phenomenological analysis method was employed to analyze the transcribed qualitative data, including identifying significant quotes and statements related to the phenomenon of student incivility; creating formulated meanings by making general restatements of the significant statements; creating theme clusters; developing an exhaustive description of nursing student incivility experiences expressed by the participants by synthesizing theme clusters and formulated meanings; identifying the essence of the phenomenon of nursing student incivility by performing a thorough analysis of the exhaustive description; and validating with the participants the final essence of nursing student incivility.

To ensure validity and trustworthiness of this research study, verbatim accounts were used to make sure that the descriptions were those of the participants. Personal biases were reflected and examined, using the bracketing technique throughout the study to set aside any biases or previous knowledge identified. Member checking was used by going back to the participants and asking them to check the precision and accuracy of their words and thoughts, and the researcher's conclusions, helping to avoid bias. An outside, experienced qualitative researcher was employed to verify the steps of this research process.

\section{Results}

The analysis provided for rich descriptions of the participants' encounters with student incivility and the effects on them personally and professionally. The encounters with incivility described by participants occurred from as recent as 2 weeks prior to the interview to as long as 14 years prior. Student incivility resulted in multifaceted tribulations for the nursing faculty members. Two main theme clusters developed related to the main research question: What is the lived experience of a nursing faculty member who has experienced nursing student incivility? The two theme clusters included: (a) frequently subjected to inappropriate student behaviors, and (b) consequences of being subjected to inappropriate student behaviors. The main theme related to theme cluster one was identified as subjected to a variety of unacceptable behaviors, primarily (a) rude and disrespectful behaviors, and (b) threatening and intimidating behaviors. Theme cluster two focused on the consequences of inappropriate student behavior, including sub-themes of (a) time consuming, (b) tarnished reputation, (c) support is beneficial, (d) harmful to health and well-being, and (e) questioning the future. Figure 1 illustrates the multifaceted tribulations of nursing faculty members who have experienced student incivility, illuminating the lived experience. 


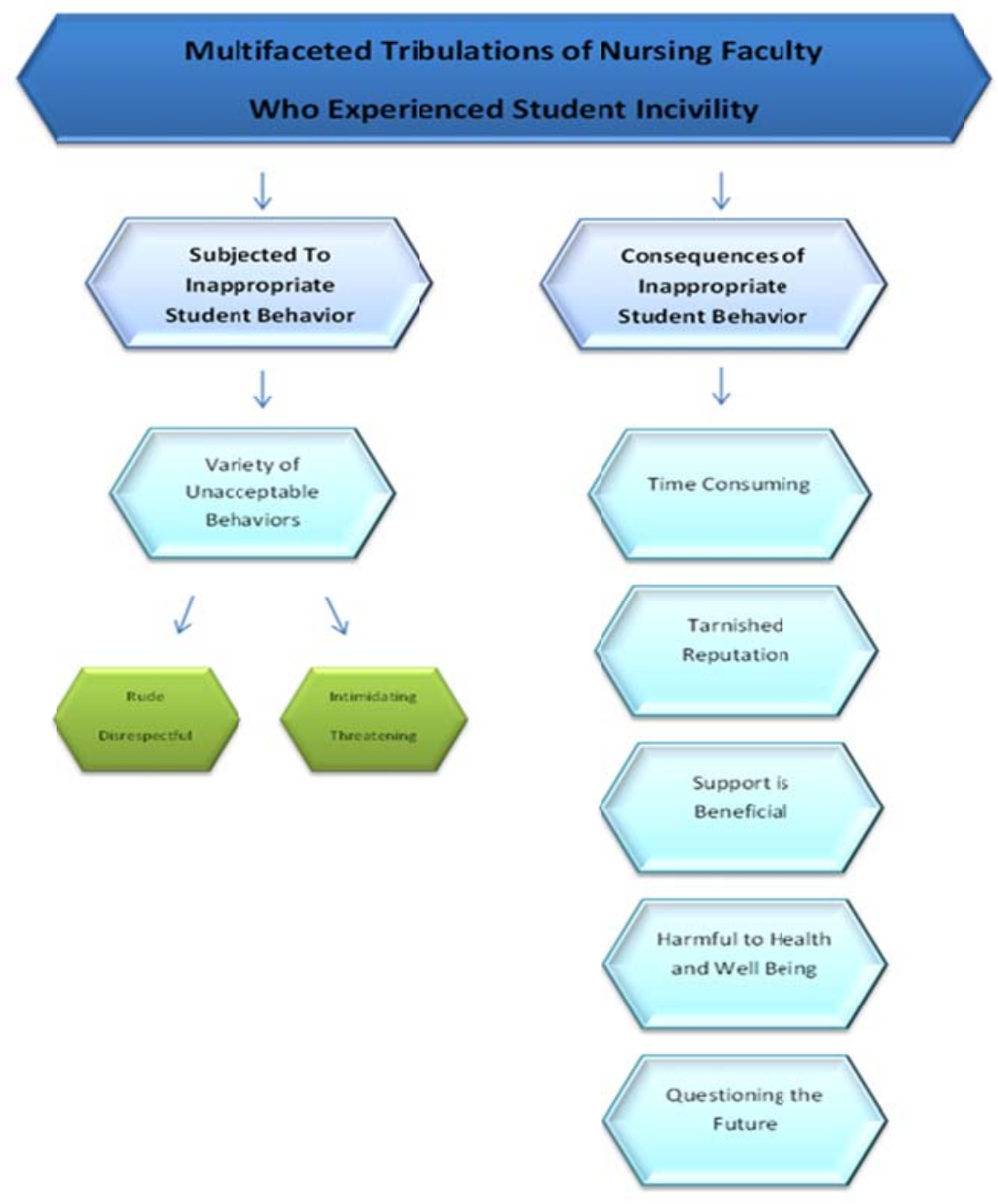

Figure 1. Multifaceted tribulations of nursing faculty who experienced student incivility

\subsection{Theme related to subjected to inappropriate student behavior}

\section{Variety of Unacceptable Behaviors}

All participants described being subjected to inappropriate and unacceptable behaviors that violate the student code of conduct. This type of student behavior ranged from rude and disrespectful behaviors such as yelling, arguing, or cheating, to threatening and intimidating behaviors such as students making threats that they will complain to administrators or a threat of potential violence towards a faculty member. Excerpts from participant interviews follow, detailing some of the unacceptable student behaviors experienced by nursing faculty.

Nancy described a meeting she had with a disgruntled student who had failed the clinical portion of the course due to unsafe clinical performance. The student exhibited not only disrespectful behavior but threatening behavior as well that caused concern.

“The student came into the office and sat down right next to me. She wouldn’t even look at me. I reviewed the reason why she was failing, and she started screaming and cussing at me. She said it was all my fault. Every word out of her mouth was profane. I physically thought she was going to hit me. She kept saying she hated me. She was angry, and I thought she was going to lash out at me.” 
Rita described vividly an inappropriate behavior that she was subjected to 14 years ago from a student who was not performing in clinical in a satisfactory manner.

"We require each week that the students have a clinical reflective diary. And in that diary he [the student] was saying how clinical is not a learning experience for him because he couldn't do anything right to please me. The line that he wrote in the diary that probably caused me the most stress was, "who do I have to kill to get you off my back?”

These behaviors were undeserving and clearly violated not only the student code of conduct but also ethical standards for nurses (and students). Nursing faculty subjected to these behaviors voiced concerns ranging from aggravation to fear.

\subsection{Themes related to consequences of being subjected to inappropriate student behavior}

\subsubsection{Time consuming}

The majority of participants noted that the time to manage or contend with uncivil student behavior was great. This increased time expenditure often took time away from the faculty member's time to prepare for teaching or other job responsibilities, leading to frustration and discontent. Extra time expenditures included activities, such as coordinating extra meeting times to discuss the situation with administration, writing counseling forms, letters of explanation, and police reports, as well as arranging for extra meeting times to counsel students. In addition, faculty noted the need to take the time to develop new policies, procedures, or methods of teaching as a result of uncivil student behavior in an attempt to prevent similar uncivil behaviors from occurring in the future. Examples follow:

Irene voiced concerns about the time she had to expend to deal with an overly disrespectful and unruly class.

"I spent more time on that class than any class I ever had. Because there were so many of them having issues with disrespectful behavior, I had to take the time to meet with all of them individually and talk with each of them about their behavior. I had to make appointments and meet with each of them in my office.”

Celeste described frustrations with having to change her class time test review policy due to an experience with severe uncivil student behavior during test reviews. This policy change requires much more time expenditure on the part of Celeste.

"I certainly changed the way I talk about exams now. I don't do exam reviews unless it is one-on-one, and we sit down in my office. I have the student e-mail me to make an appointment. The student comes to my office, and we do the exam review in my office. Of course, this is more time consuming for me, but helps prevent this rude behavior."

\subsubsection{Tarnished reputation}

Damage to the faculty member's reputation was a major concern and consequence of incidences of student incivility towards nursing faculty. Both personal and professional attacks were made to most faculty members' reputations. Social media, circulation of letters, student comments on faculty evaluations, and word-of-mouth were all avenues for attempting to damage the faculty members' reputations. Faculty described this as upsetting and hurtful.

Theresa was distressed that an uncivil student was attempting to harm her professional reputation by making negative comments about her on Facebook.

"Other students were telling me that there was a Facebook posting like an ongoing site where she [the uncivil student] was saying things that were uncomplimentary about her experiences with me, and it was not only 
directed towards me but her behavior was directed at other students. She was trying to harm my reputation with other students. The postings were among student groups, so definitely she was trying to harm my professionalism."

Laura was extremely upset by the behavior of a group of uncivil students in her class that were spreading rumors around to the class to try to discredit her.

“It was that they were sending e-mails around to classmates to report me to the board of nursing. I don't know what they thought I was violating, but they were telling the class they should file a complaint against me to the board of nursing. They were badmouthing me, especially one gal in particular. She began telling other students that they had gotten me fired, that I was losing my job, that I had been demoted from teaching in the classroom, and I would be losing my job at the end of my contract, and I had been disciplined. All these things were not true. It was an attempt to discredit, diminish my role, and ruin my reputation. Basically to undermined me as a faculty."

\subsubsection{Support is beneficial}

The importance of administrative, peer, family, or security support was noted by all participants. Faculty found this to be very beneficial when dealing with incivility. Others who experienced a lack of support found this troubling and voiced a strong need. Sample descriptions of participants' thoughts are below.

Isabelle found support from security and administration comforting after receiving a threatening letter in the mail from a student. Isabelle recalled:

"The police took it very seriously and actually did check the return address and found out who it came from. The dean also responded to a letter I wrote her requesting the doors to our office suite remain locked. The dean said they would be kept locked. The dean reassured me that the suites would be kept locked. This made me feel a bit safer."

Laura discussed how the lack of support made her feel.

"I kind of feel like almost defeated. I felt almost victimized that it was allowed to go on without repercussion, and I felt faculty are almost a sitting target. You know that students can do this [act uncivil], and faculty can file formal complaints and nothing is going to happen. So I feel here at this college that students are allowed to do it [act uncivil]. I really felt helpless and that is not a good feeling for me to have.”

All participants valued being supported by their superiors, peers, and other pertinent people. The support led to a feeling of being defended, validated, and important. In contrast, participants who were not supported felt disappointed, hurt, and unimportant.

\subsubsection{Harmful to health and well-being}

All participants described being harmed emotionally and/or physically. Participants voiced being scared, worried, intimidated, threatened, paranoid, stressed, distressed, upset, defeated, and sad. Some described feelings of anxiety and dread. Several participants reported feeling upset due to the classroom disruption resulting from incivility and the negative impact the uncivil behavior had on their relationship with a student or group of students. Participants described negative physical effects such as migraines, bowel disorders, inability to sleep, and crying.

Rita reported being scared and feeling like her family was in danger after receiving a threatening note directed towards her and her family in a student diary (even 14 years later). 
"I was concerned about my family. I had small children and a husband. My phone number is in the book. And we live in a small community. It wouldn't take much to look through the telephone book and find my name. My concern was that he [the student] was saying who 'would he have to kill to get me off his back.' My first thought was that he would harm my family-come after my family. I wasn't as concerned about me as I was my family. For a long time we [my family] didn't really answer our phone. We would let the machine take it. And at the college, I had caller ID [identification] and I would watch to see who was calling before picking up the phone. I would listen to my messages to see who called. I was very cautious about not having contact with him [the student].”

Laura, who was subjected to an extremely uncivil group of students, illustrated the magnitude of the physical, emotional, and psychological consequences she experienced.

"It caused anxiety, tearfulness. I spent days where I was just crying because I did not want to come back to work. This is not why I am teaching, this is not why I am here. I almost felt helpless. I felt defeated. Just the anxiety of having to deal with these people in this class and not having anyone else to teach with is kind of like you are flapping out there in the breeze by yourself to manage all this, so I was pretty spent by the end of the semester. I was done. I also felt scared, physically threatened. I felt the potential was there. I felt there was a very real potential that one could be physically violent towards me. I was not sleeping well. I was losing sleep. I was crying. I started getting migraines and I have not had migraines in years and I started getting migraines again. I had GI [gastrointestinal] upset almost like irritable bowel syndrome symptoms. So yes, I had many physical consequences from dealing with this experience.”

\subsubsection{Questioning the future}

All participants questioned their desire to continue teaching after experiencing uncivil student experiences. Although they did question the possibly of leaving education, most voiced a desire to continue to teach due to their love of teaching. The possibility for leaving education was seriously contemplated by some.

Ida noted the uncivil student behavior made her question continuing teaching.

"I love to teach. I was told by many that I should teach. But I did really think, "is this what I want to do or should I quit?” But I have worked too hard to quit. I have had those thoughts about quitting, but I keep coming back and something refreshes me. So yes, I keep plugging away.”

Nancy also questioned her desire to continue teaching after an uncivil encounter.

I almost didn't teach clinical anymore after that [uncivil encounter] because it was so stressful for me. I had a bad taste in my mouth for a while and I didn't want anything to do with clinical for a while. But after you have some time off, you come back and have a different perspective. You have to think of it as having really nice and good students most of the time. In cases like this, though, it does make you think, is this what I want to do?”

\subsubsection{Exhaustive description}

The experience of nursing student incivility for nursing faculty members is multifaceted. Resulting tribulations were described as disrespectful, rude, frustrating, intimidating, threatening, frightful, upsetting, time consuming, and harmful, both physically and emotionally. All nursing faculty described being subjected to rude, discourteous, and disruptive student behaviors in the classroom, in clinical, and in their faculty offices. Feelings that resulted from this uncivil behavior included shock, anger, worry, fright, and distress, often lasting for an extended period of time. Uncivil student treatment often damaged reputations and hindered the effectiveness of the learning environment as well as the teacher-student relationship. A support system was perceived by the nursing faculty as necessary and comforting, providing needed 
assistance and encouragement. Although faculty members questioned their desire to remain in teaching, the resounding consensus from the majority of participants was a continued passion for teaching.

\section{Discussion}

Incivility is occurring in the academic setting and nurse educators are experiencing this in the nursing educational environment. This troubling phenomenon is contrary to the essence of nursing. Nursing is based on the concept of caring. Although the nursing educational environment should be based on mutual respect and civility, this is not always the case. As evidenced by participants' stories, nursing faculty members in this study were commonly subjected to uncivil and unacceptable student behaviors, risking personal emotional and physical harm to the faculty members.

A majority of participants described being very concerned over damage to their professional reputation as a consequence of student incivility, a theme that has had limited discussion in the nursing literature. This slanderous and discrediting damage to participants' reputation was often carried out in a very public arena on social media sites, for example, Facebook, or on public websites that rate a professor's job performance, such as Rate My Professors. Clark and Springer ${ }^{[22]}$ explored issues of academic incivility and found that faculty often were subjected to being publicly criticized and badmouthed by students in an attempt to discredit the faculty member. Misuse of social media is becoming common today, with cyberbullying receiving much national attention ${ }^{[35]}$. The American Nurses Association and the National Council of State Boards of Nursing have implemented social media policies for nurses and nursing students. Engaging in unethical and unprofessional conduct aimed at damaging one's reputation via social media is not acceptable and could be viewed as lateral violence ${ }^{[36]}$.

The importance of administrative and peer support is noted in the nursing literature sparingly. Nurse scholars who have examined academic incivility have reported enforcing a strong student code of conduct may offer support for faculty members with managing student incivility. Many nurse researchers have identified that the rigors of nursing education are stressful, and often lead to behavioral changes, including incivility ${ }^{[14-16]}$. These findings demonstrate the need for teaching students stress management strategies during their educational program to manage and reduce stress. In addition, training in conflict management skills for both students and faculty can play a critical role in success ${ }^{[7,10,22]}$.

Nurse researchers have found that nursing student incivility has resulted in nursing faculty resigning, retiring, or withdrawing from their teaching positions ${ }^{[8,9,37-39]}$. Although this was not a conclusion in this study, the intensity and severity of some of the uncivil acts could certainly justify faculty loss. This trend noted in the nursing literature as a result of student incivility is troubling. There is already a shortage of qualified nurse educators. The nursing profession cannot afford to lose nurse educators as a result of student incivility.

\subsection{I mplications for nursing education}

Those joining the nursing profession must demonstrate and value the underlying principles of caring and respect that are essential for nurses to embrace. Accordingly, in the nursing educational environment, the student-teacher relationship should be based on respect and care. It is very disturbing to contemplate that uncaring and disrespectful behaviors exhibited by students while in nursing school may very well extend into the workplace once the student graduates. Therefore, developing and implementing effective policies and procedures to prevent and manage student incivility in the nursing education setting is suggested. In addition, effective communication, faculty role modeling, and education for students regarding incivility issues are necessary. Furthermore, faculty members subjected to student incivility should receive the support and guidance needed to manage incidences of student incivility.

\subsection{Development of policies and procedures}

This study clearly demonstrates the need for colleges to consider the development or revision of code of conduct policies for both students and faculty members outlining expectations for civility and consequences of violating the policies. 
Colleges must have no tolerance or lenience for acts of incivility exhibited by students or faculty members. These policies must be very visible, transparent, and easily accessible for all to view. In addition, it is suggested that faculty members include these policies in their syllabi and review the policies with students at the beginning of each semester ${ }^{[7,10,40]}$.

\subsection{Student development}

This study demonstrates the need for students to understand and internalize the value and importance of civility, caring and respect from day one of their college experience. Faculty role modeling civil behavior is a valuable method to promote and establish a culture of civility in the academic setting. Faculty must lead by example and demonstrate respect and caring behaviors in all interactions with students and peers. This will, in turn, allow students to identify the expectations for a culture of civility ${ }^{[10,40]}$. Throughout the nursing program, students should be taught about professional and ethical codes of conduct. Dialogues with students focusing on treating patients, families, peers, and co-workers with respect and care are important ${ }^{[40]}$. Student education regarding the importance of establishing a culture of civility in the educational setting while in nursing school may also help to establish expectations and a demand for a civil work environment.

\subsection{Faculty development and support}

In the nursing education setting, it is imperative for administration and fellow colleagues to provide support and encouragement for nursing faculty members who have experienced student incivility. Supportive behaviors such as listening and mentoring exhibited by administrators may help an individual who has experience student incivility to have a sense of feeling important, valued, and respected. In addition, administrators should provide information for faculty on prevention strategies, as well as suggestions on how to intervene and handle an uncivil encounter. Offering faculty development specifically addressing the topic of student incivility may help faculty feel empowered and better able to effectively prevent or manage student incivility ${ }^{[10]}$.

\subsection{Limitations}

Limitations include the fact that only experiences and perceptions of nursing faculty members who experience student incivility were examined. This presents a narrow and limited understanding of the larger and more encompassing phenomenon of academic incivility. Additionally, the participants interviewed were all Caucasian, female faculty members. Interviewing male faculty or ethnic/racial minority faculty members may have yielded different findings. Furthermore, the accuracy of the information gathered from the participants during the interview process cannot be absolutely established. In self-report research studies, participants may not always be truthful, may omit information, or may exaggerate the facts.

\subsection{Recommendations for future research}

There are numerous opportunities for further research on the topic of academic incivility in nursing education. Examples of nursing student incivility are increasing. The far-reaching effects this incivility has on nursing faculty has had limited investigation. Pursuing further research exploring the lived experience of nursing faculty members who have experienced student incivility may provide a more thorough understanding of this phenomenon. Development of a quantitative study to investigate this topic, using the emerged themes from this current study, would allow for a large sample size with greater geographical representation to be reached, helping to provide further understanding of this phenomenon.

Further research might explore the characteristics of the initiator and recipient of the incivility. For example, does age, gender, or status affect the type or frequency of uncivil encounters? Further studies may examine contributing factors to student incivility in nursing education, such as the role of stress, generational differences, and attitudes of entitlement. This information may lead to valuable ideas for management and prevention of student incivility.

Further research is needed to explore the valuable benefit support for nursing faculty members who have experienced student incivility offers. Exploring if the effectiveness of support is determined by the type of person giving the support 
may provide valuable information. For instance, is support more effective if given by an administrator, a peer, or a family member?

Incivility in nursing education is not limited to student-to-faculty incivility. Exploring faculty-to-student incivility and incivility between faculty and administrators is also an important avenue to pursue. All of these types of incivility damage the overall effectiveness of the nursing educational setting. Information obtained through research may prove beneficial for reducing or eliminating academic incivility.

\section{Conclusion}

Findings from this current study clearly support findings from studies found in the nursing literature, as well as the larger body of research on organizational workplace incivility. This information strongly suggests the importance of identifying contributing factors of incivility present in nursing students and implementing policies to deal with incivility. Themes that emerged from data analysis provide insights into the variety of uncivil student behaviors that occur along a continuum that nursing faculty members are subjected to, as well as adverse physical, psychological, and emotional effects that experiences of nursing student incivility have on nursing faculty members. Additionally, details of how these experiences affect the teaching-learning process and the academic environment are illuminated. Findings support the view that nursing student incivility is becoming more prevalent on college campuses and can have devastating effects on nursing faculty members.

\section{References}

[1] Taylor C, Bator S, Hull E, Hill JJ, Spurlock W. Incivility: The antithesis of caring. In J. T. Catalano (Ed.), Nursing Now! Today’s Issues, Tomorrow's Trends (6th ed., pp. 297-309). Philadelphia, PA: F. A. Davis, 2012.

[2] Cortina LM, Magley VJ, Williams JH, Langhout RD. Incivility in the workplace: Incidence and impact. Journal of Occupational Health Psychology. 2001; 6(1): 64-80. http://dx.doi.org/10.1037/1076-8998.6.1.64

[3] Felbinger DM. Bullying, incivility, and disruptive behaviors in the healthcare setting: Identification, impact, and intervention. Frontiers of Health Services Management. 2009; 25(4): 13-23.

[4] Heinrich KT. Joy stealing 10 mean games faculty play and how to stop the gaming. Nurse Educator. 2007; 32(1): 34-38. http://dx.doi.org/10.1097/00006223-200701000-00010

[5] Johnson PR, Indvik J. Rudeness at work: Impulse over restraint. Public Personnel Management. 2001; 30(4): $457-465$.

[6] Clark C. The dance of incivility in nursing education as described by nursing faculty and students. Advances in Nursing Science. 2008a; 31(4): E37-E54. http://dx.doi.org/10.1097/01.ANS.0000341419.96338.a3

[7] Suplee PD, Lachman VD, Siebert B, Anselmi KK. Managing nursing student incivility in the classroom, clinical setting, and on-line. Journal of Nursing Law. 2008; 12(2): 68-77. http://dx.doi.org/10.1891/1073-7472.12.2.68

[8] Clark CM. Faculty and student assessment of experience with incivility in nursing education. Journal of Nursing Education. 2008b; 47(10): 458-465. http://dx.doi.org/10.3928/01484834-20081001-03

[9] Luparell S. The effects of student incivility on nursing faculty. Journal of Nursing Education. 2007; 46(1): 15-19. PMid:17302095

[10] Clark CM, Springer PJ. Academic nurse leaders' role in fostering a culture of civility in nursing education. Journal of Nursing Education. 2010; 49(6): 319-325. http://.dx.doi.org/10.3928/01484834-20100224-01

[11] Clark CM, Kenaley BLD. Faculty empowerment of students to foster civility in nursing education: A merging of two conceptual models. Nursing Outlook. 2011; 59: 158-165. http://dx.doi.org/10.1016/j.outlook.2010.12.005

[12] Clark C. Continuum of incivility. 2009a. Retrieved from http://hs.boisestate.edu /civilitymatters/resources.pubs.htm

[13] Clark CM. Student voices on faculty incivility in nursing education: A conceptual model. Nursing Education Perspectives. 2008c; 29(5): 284-289. PMid:18834058

[14] Gibbons C, Dempster M, Moutray M. Stress and eustress in nursing students. Journal of Advanced Nursing. 2008; 61(3): $282-290$. http://dx.doi.org/10.1111/j.1365-2648.2007.04497.x

[15] Shirey MR. An evidence-based solution for minimizing stress and anger in nursing students. Journal of Nursing Education. 2007; 46(12): 568-571. PMid:18196841 
[16] Tully A. Stress, sources of stress and ways of coping among psychiatric nursing students. Journal of Psychiatric and Mental Health Nursing. 2004;11: 43-47. http://dx.doi.org/10.1111/j.1365-2850.2004.00682.x

[17] Beck DL, Hackett MB, Srivastava R, McKim E, Rockwell B. Perceived level and sources of stress in university professional schools. Journal of Nursing Education. 1997; 36(4): 180-186. PMid:9107596

[18] Robertson JE. Can’t we all just get along? A primer on student incivility in nursing education. Nursing Education Perspective. 2012; 33(1): 21-26. http://dx.doi.org/10.5480/1536-5026-33.1.21

[19] Mahat G. Stress and coping: First-year Nepalese nursing students in clinical settings. Journal of Nursing Education. 1996; 35(4): 163-169. PMid:8830130

[20] Pagana K. Stresses and threats reported by baccalaureate students in relation to an initial clinical experience. Journal of Nursing Education. 1988; 27(9): 418-424. PMid:2852715

[21] Pagana K. The relationship of hardiness and social support to student appraisal of stress in an initial clinical nursing situation. Journal of Nursing Education. 1990; 29(6): 255-261. PMid:2165145

[22] Clark C, Springer PJ. Thoughts on incivility: Student and faculty perceptions of uncivil behavior in nursing education. Nursing Education Perspectives. 2007; 28(2): 93-97. PMid:17486799

[23] Lasiter S, Marchiondo L, Marchiondo K. Student narratives of faculty incivility. Nursing Outlook. 2012; 60: 121-126. http://dx.doi.org/10.1016/j.outlook.2011.06.001

[24] Lashley FR, DeMeneses M. Student civility in nursing programs: A national survey. Journal of Professional Nursing. 2012; 17(2): 81-86. http://dx.doi.org/10.1053/jpnu.2001.22271

[25] Clark CM. National study on faculty-to-faculty incivility: strategies to foster collegiality and civility. Nurse Educator. 2013; 38(3): 98-102. http://dx.doi.org/10.1097/NNE.0b013e31828dc1b2

[26] Clark CM, Olender L, Kenski D, Cardoni C. Exploring and addressing faculty-to-faculty incivility: A national perspective and literature review. Journal of Nursing Education. 2013; 52(4): 211-218. http://dx.doi.org/10.3928/01484834-20130319-01

[27] Heinrich KT. Joy-stealing games. Reflections on Nursing Leadership. 2006; 32(2): 1-4.

[28] Clark CM, Carnossa J. Civility: A concept analysis. Journal of Theory Construction \& Testing. 2008; 12(1): 11-15.

[29] Creswell, JW. Qualitative Inquiry \& Research Design: Choosing Among Five Approaches (2nd ed.). Thousand Oaks, CA: Sage, 2007.

[30] Flood A. Understanding phenomenology. Nurse Researcher. 2010; 17(2): 7-15. http://dx.doi.org/10.7748/nr2010.01.17.2.7.c7457

[31] Mapp T. Understanding phenomenology: The lived experience. British Journal of Midwifery. 2008; 16(5): 308-311.

[32] McConnell-Henry T, Chapman Y, Francis K. Husserl and Heidegger: Exploring the disparity. International Journal of Nursing Practice. 2009; 15: 7-15. http://dx.doi.org/10.1111/j.1440-172X.2008.01724.x

[33] Starks H, Trinidad SB. Choose your method: A comparison of phenomenology, discourse analysis, and grounded theory. Qualitative Health Research. 2007; 17(10): 1372-1380. http://dx.doi.org/10.1177/1049732307307031

[34] Colaizzi P. Psychological research as the phenomenologist views it. In R. S. Valle \& M. King (Eds), Existential Phenomenological Alternatives for Psychology (pp. 48-79). New York, NY: Oxford University Press, 1978.

[35] Weber Shandwick. Civility in America 2012. 2012. Available from: http://www.webershandwick.com/civility/

[36] National Council of State Boards of Nursing. White paper: A nurse's guide to the use of social media. 2011. Available from: www.ncsbn.org/Social_media_guidelines.pd

[37] Altmiller G. Student perceptions of incivility in nursing education: Implications for educators. Nursing Education Perspectives. 2012; 33(1): 15-20. http://dx.doi.org/10.5480/1536-5026-33.1.15

[38] DalPezzo NK, Jett, KT. Nursing faculty: A vulnerable population. Journal of Nursing Education. 2010; 49(3): 132-136. http://.dx. doi.org/10.3928/01484834-20090915-04

[39] Luparell S. Incivility in nursing: The connection between academia and clinical settings. Critical Care Nurse. 2011; 31(2): 92-95. http://dx.doi.org/10.4037/ccn2011171

[40] Clark CM. Faculty field guide for promoting student civility in the classroom. Nurse Educator. 2009b; 34(5): 194-197. http://dx.doi.org/10.1097/NNE.0b013e3181b2b589 